\title{
IMAGE BASED WEATHER AND TEMPERATURE ANALYSIS SYSTEM
}

\author{
Ashlin Francis \\ Department of Computer Science \\ RIT, Kottayam, Kerala, India
}

\author{
Vineetha S \\ Department of Computer Science \\ RIT, Kottayam, Kerala, India
}

Abstract-Weather prediction is always a hot topic, as it has great importance in many fields. Recent issues related to climate change shows the importance of weather in man's daily life. Weather information has a big social impact. There are a lot of methods to predict weather conditions but most of them use additional hardware. For an example, weather prediction using separate weather satellites. These methods are very expensive. This is the place where image based weather recognition is important. Image based weather recognition is an emerging topic. Images carry a lot of information to understand weather. The main advantage of image based weather recognition is large quantity of data, compare to other methods it doesn't need additional hardware, comparatively less cost and images are easily accessible. Image based weather recognition has large applications like driving assistance, robotics etc. Convolutional neural network, a deep learning method is used to identify the weather information. Weather is divided in to six classes: cloudy, haze, rainy, snowy, sunny, thunder. Images are considered as clusters and each cluster is considered for feature extraction.

Temperature analysis is the other field in this work. Even though temperature is influenced by many factors like humidity, pressure, weather etc. Weather has a great influence on temperature. Temperature analysis is calculated using the scores obtained in each weather classes.

Keywords-Convolutional Neural Network , VGG16, Euclidean Distance, Image based Weather Recognition.

\section{INTRODUCTION}

Weather prediction always had a great importance in science and technology. It has a great importance from the day man started to think. Ancient days people did informal methods to predict weather. Now we depend on science and technology to predict weather. Weather is a hot topic all the time as it has many applications like agriculture, military purpose. There are a lot of methods to predict weather conditions but most of them use additional hardware. For an example, weather prediction using separate weather satellites.
These methods are very expensive. This is the place where image based weather recognition is important.

Image based weather recognition is an emerging topic in computer vision. Weather recognition is a difficult area compared to other object recognition methods. It is difficult to understand phenomena like lightening and reflection on images. Weather has vital role in daily lives. Weather information has a huge social influence. Availability of images makes it more easy to access the data and image based weather recognition does not require additional hardware so this makes it less expensive. Another advantage is large quantity of image can be used to analyze the weather. Imagebased weather recognition has many applications. One of the example is solar energy system, the amount of power obtained in an area can be calculated. Driving assistance can be provided from the instant response. Image based weather recognition has an inevitable role in robotics, wireless sensor networks, remote sensing areas.

Weather recognition from image is a difficult task. In order to improve the accuracy it is better to use different scenes. Here consider diff erent scenes to identify the weather condition. Image based weather recognition is difficult compare to other object detection as it has no specific feature to identify weather condition. Every weather condition recognized using diff erent features. For example shadows help to recognize sunny class. So here we use convolutional neural network. Using cnn we try to analyse the weather condition of the image. Here weather is divided in to six classes: cloudy, haze, rainy, snow, sunny, thunder. Convolutional neural network is a part of deep learning. It uses neurons for learning. Neuron learns to convert input signal into corresponding output signal. Cnn contains four layers. Convolutional layer do operations on the features. Relu layer take the value above a threshold value. Pooling layer calculate maximum value in each and connected layer mainly used for classification. 


\section{International Journal of Engineering Applied Sciences and Technology, 2019 Vol. 4, Issue 2, ISSN No. 2455-2143, Pages 59-66 \\ Published Online June 2019 in IJEAST (http://www.ijeast.com)}

Temperature analysis is the another concern of this project. Temperature is influenced by many factors and one of the important factor is weather. Here score obtained from weather analysis used to analyse temperature.

\section{A. Motivation}

Image based weather recognition is emerging topic in weather recognition. There are many weather recognition methods. These methods needs additional hardware and they are expensive. Image based weather recognition has many advantages. Easy accessibility of dataset, no additional hardware needed are some of them. But to identify weather condition from an image is a challenging task. The reason is that there is no specific feature to identify weather condition. We need to consider different features while considering diff erent weather classes.

\section{B. Objective}

The main objective is to develop a tool to obtain weather information from an image using cnn. Objective includes:

- To detect the weather condition from the image using cnn for multiple classes: cloudy, haze, snowy, sunny, rainy, thunder.

- To identify the approximate temperature from the scores obtained in each classes.

The rest of the paper is organized as follows. Related works are explained in section II. The proposed system is presented in section III. Experiment results are given in section IV. Screenshots are provided in section V. Future work and application are provided in VI. Concluding remarks are given in section VII.

\section{RELATED WORK}

In this section we discuss some of the weather recognition techniques. There are many weather recognition methods. Early days, most of the methods needed additional hardware. But growth in computer vision made a great change in traditional ways of weather recognition. Some of the earlier works on image based weather recognition had interesting ideas but the accuracy of these methods are very low. Roser et.al(2008)[9]proposed a method to improve machine vision in bad weather situations for driving assistance. In this method SVM classifier is used. In the method, Yang et.al(2015)[4] and Wang et.al(2010)[7] uses spatial pyramid matching for classification of images. Wang et.al[7] improves spatial pyramid matching using locality linear coding. Yan et al.(2009)[13] put forward an approach to identify weather information from road images. It uses adaboost algorithm for classification. Development in deep learning brought a great positive change in Image based weather recognition. Lu et al.(2017)[1] developed a system to classify cloudy and sunny images. Method used here deep learning and SVM used for classification. Din lin et.al.(2017)[2] uses two modules, region selection and concurrency for image based weather recognition. Separate cnn architecture used in both modules. Elhoseiny et.al(2015)[5] uses a typical cnn method to classify images in to two classes: sunny and cloudy. Zhang et al.(2015)[8] put forward a method to multi-class weather classification. Features are classified for sunny, rainy, snowy and haze weather. Multi-kernel learning uses predefined features to recognize weather condition. Some of the methods are described briefly below.

\section{A. Image Based Weather Recognition tools using Deep Learning}

Compare to other object detection method, weather detection is much difficult but development in computer vision made it possible. Most of the methods mainly focused on some weather conditions and limited to certain classes. In some methods to identify each features separate learning methods are used. This increase its complexity.

Two Class Weather Classification(2017)[1] classify the images in to two classes: sunny or cloudy. It is restricted to limited images. In this method along with cnn features. It also taken the advantage of human vision. People understand weather using sky, haze, shadow etc features. These features are called weather features. Weather features obtained by combining data driven cnn feature and well chosen weather specific features. Here features like sky, shadow, reflection, contrast, haze is considered. Hazy and grayish sky detects cloudy day. Dark shadows maps to sunny day. For making training set more useful it uses a novel approach. Din lin et.al(2017)[2] uses two modules :region selection and concurrency for image based weather recognition. It divides each images in to regions and each region is considered. It uses deep learning method. An additional Siamese architecture is used in concurrency module which make it more complex. It uses cosegmentation method for feature extraction. As it considers each regions computational complexity is high. Each module use separate cnn architecture.

Weather Classification using deep convolutional networks(2015)[5] uses a typical cnn method to classify images in to two classes:sunny and cloudy. The cnn architecture contains eight layers. It contain five convolutional or pooling layers and three fully connected layer.It used a model inspired by Krizhevzky et.al(2012)[26] imagenet cnn. It does not consider any other weather features. In this case the problem is it may not be used for a large variety of images dataset.

J. Dai, K. He, and J. Sun(2016)[3] uses a convolutional feature masking for feature extraction. Semantic segmentation is used to identify object instances. Here instance aware semantic segmentation is used. It is done through three 
diff erent and related subtask.1) Diff erentiating instances using bounding boxes. Region proposal network used to predict location and objectness scores. 2) a pixel level mask is predicted for each instances. 3)categorizing instances. For each stages it uses convolutional neural network.

Multiclass Weather Classification of single images(2015)[8] is a method for image based weather recognition. Here only four classes are considered. Multiple weather features are considered for each classes. Classes are sunny, rainy, snowy and haze. To provide scores to each class, it consider diff erent features for diff erent classes. To identify the sunny feature of the image, it consider clear sky and strong shadows. To identify rainy feature, it uses HGG based template matching. Snow feature is treated as noise and pixels contain snowflake have a grey value greater than threshold value. For haze feature intensity of pixels is considered. As for each class diff erent features are considered, it uses multiple kernal learning method.

\section{B. Image Based Weather Recognition methods using other Soft Computing Algorithms}

case Im

Image based weather recognition using other soft computing algorithms are given below. The main limitation of these methods are the accuracy of these methods are very less compare to deep learning tools. Linear spatial pyramid matching(2015)[4] is a method using linear coding in traditional spatial pyramid matching method. In SPM, for coding linear method is used. It considers local constraints then euclidean distance calculated and weights are adjusted. In state of art image classification systems contains bag-offeatures and spatial pyramid matching. The Bag of features does not consider spatial layout, so it is not suitable for capturing shape or detecting image. So SPM is used. First, feature points are detected and this form descriptor layer then code layer used for coding. Vector quantization is used for generating histograms. Then this histograms are used to give final output.

Wang et.al(2010)[7]proposed linear classification in spatial pyramid matching. SPM is described in above paragraph. In this method instead of vector quantization, it uses locality linear constraint coding. It uses locality constraint instead of sparsity constraint. The advantages of locality constraint are better reconstruction, local smooth sparsity etc. Classification of weather situations on single color images(2008)[9] is a method to improve machine vision in bad weather situations for driving assistance. It classify images in to clear sky, light rain, heavy rain. SVM method is used to classification. It choose ROI using features: local contrast, minimum brightness, sharpness hue and saturation. Based on it descriptor developed and applied SVM for classification. It works on single monoscopic color image.

\section{Temperature Prediction}

Temperature prediction from the analyzed weather condition is the secondary task of the project. Among many visual attributes, weather attribute is one of the important attribute. Temperature is influenced by many factors and weather is one of the main factor. Wei et.al(2018)[10] developed a system to identify temperature condition from an image. Temperature range is divided in to 72 classes. Visual features of images are extracted using cnn . Corresponding to each image it contains a 72 vector. In training phase the temperature of image is mapped to a particular class by giving one to that class and all other classes contain value zero. Here base architecture of cnn used from scratch based on Skyfinder dataset. It contains four convolutional layers which is followed by four fully connected layers. Output of the model is a 70D vector indicating the probabilities of diff erent temperatures.

\section{METHODOLOGY}

Image based weather recognition is a challenging task.Compare to other object detection it does not have a particular feature to identify the weather condition. For an example,sunny feature is identified by shadow of buildings, intensity of light etc and features used for other classes will be diff erent. Due to variations in features, convolutuional neural network is used. For that system contain two phases :

1. Training phase:-to train the images of different classes.Here input is dataset of images of diff erent classes. Output is a file that contain features of the image and label to which class it belongs.

2. Testing phase:- Here image is given as input and output is the scores to diff erent weather classes.

Main idea of the project is to develop a tool to identify the weather condition from an image. Project comprises following tasks. In pre-processing stage, images are divided in to clusters using Kmeans algorithm. For feature extraction VGG16 model is used as base model. Concurrency between the clusters are calculated using Euclidean distance. SGDC used for system learning. Using decision function the scores to each class for an image is obtained. For temperature calculation scores obtained in each class is used to provide weights to each classes. The figures 1 and 2 depicts the block diagram of training and testing phase.

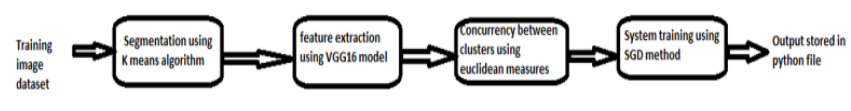

Fig. 1. The block diagram of training phase 


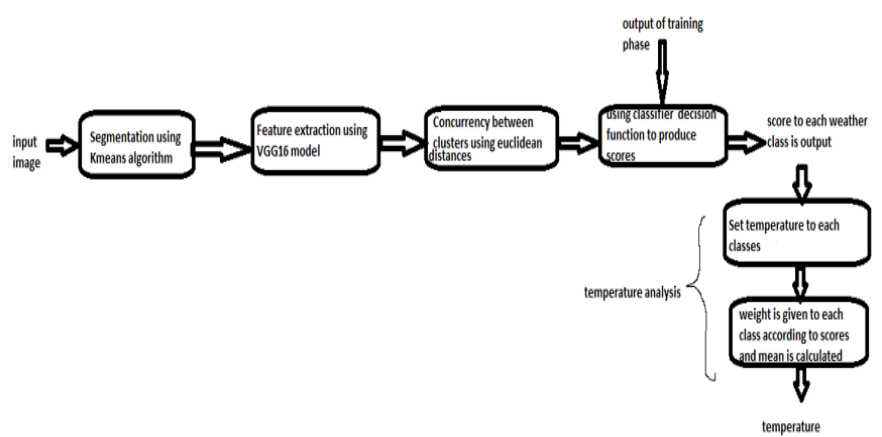

Fig. 2. The block diagram of testing phase

\section{A. Dataset}

In training phase, dataset is important. Here we use weather dataset contain 200 images for each six classes: sunny,cloudy, rainy, snowy, haze and thunder.Images are collected from google,explorer etc.The 1200 images are divided in to six classes. The dataset for weather classification is very important. Each image of diff erent classes is critical. Weather condition of each image is identified. Single weather class is labeled to each image in training set. This helps in classification and analyze the weather condition.

\section{B. Training Phase}

Training phase trains image of diff erent weather classes. Here consider six weather classes: cloudy, haze, rainy, snow, sunny, thunder. It has different stages . First stage is segmenting images. Second stage is for feature extraction. Third stage helps to find coexistence between clusters. System training is done using SGDC method.

\section{Image segmentation}

In segmentation stage, image is divided in to clusters. Kmeans clustering is used for clustering. Here number of clusters is seven. So images are divided in to clusters. Each clusters are given to feature extraction.For representing each images belongs to particular class in training phase, first row during feature extraction is used for representing classes. 0 represents cloudy class, 1 represents haze class, 2 represents rainy class, 3 represents snow class, 4 represents sunny class and 5 represents thunder class. Figure 3 shows the example of image segmentation.

\section{Feature Extraction}

The main challenging task is feature extraction.Unlike other object detection, weather detection does not use a particular feature. So here used cnn model to extract features of image.VGG16(2014)[16] is the base model used. It contain 16 layers. This model is used for object detection. In training phase it store the unique features of the image.Convolutional layer, pooling layer and relu layer used to generate feature. Fully connected layer give final output. Here VGG16 used as base cnn architecture and output of second fully connected layer is taken as output. It contain 4096D values representing the image. As each image divided in to seven clusters, a image is represented by $7 * 4096$ matrix. Each row specify the features of each cluster.

If $I$ represents image, seg represents segments, $k$ represents number of clusters then region feature of a segment, $f$ is

$$
F=G(\Theta ; I ; s e g ; k)
$$

Here $G(\Theta)$ represents architecture of cnn.
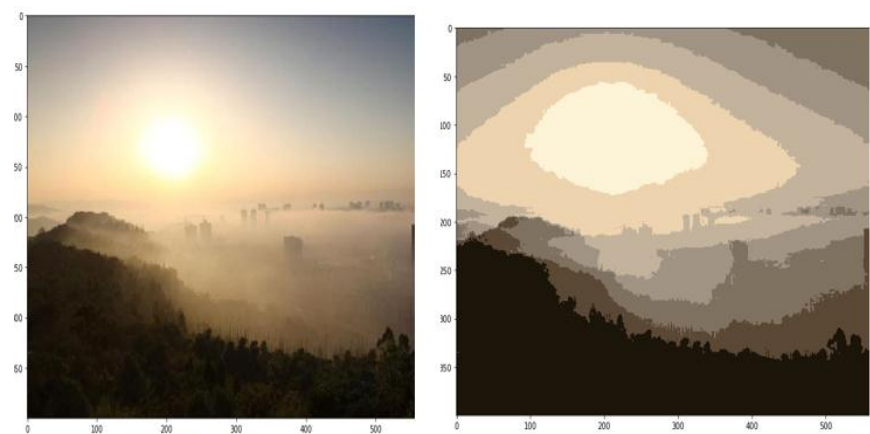

Fig. 3. Figure shows the example of segmentation

\section{Concurrency of segments}

This module uses to find the corelation between the segments. To improve the accuracy, concurrency between the clusters are calculated. In some cases the coexistence of certain segments helps to identify weather condition. Euclidean distance is calculated for finding the similarity between the cluster features.

If $F_{i}$ and $F_{j}$ represent features of clusters $i^{\text {th }}$ and $j^{\text {th }}$ respectively then feature concurrency,

$$
F_{c}=\text { euclideandistances }\left(F_{i}, F_{j}\right)
$$

\section{Complete Decision Function}

Here features of each clusters and the similarity between the features is considered. So the complete decision function will be

$$
\text { Featureofimage, } F_{i}=\left(F, F_{c}\right)
$$

For system learning SGDC method used. It is an optimization algorithm. Using class decision function, scores are provided to each class. 


\section{Testing Phase}

In testing phase, input is a outdoor image. Output is scores obtained for diff erent weather classes. Weights are obtained from this scores for temperature analysis.First three stages of testing phase is similar to training phase.Here input image is divided in to cluster and feature of image is extracted. Difference is in weather analysis and temperature analysis stages.

\section{Weather Analysis}

In weather analysis, the data file from training phase is used to calculate the scores for each class. Here class decision function is used to get the scores.Output of this stage will produce values to diff erent class based on the weather condition of that image.

\section{Temperature Analysis}

Temperature is influenced by many factors. One of the main factor is weather. Once weather condition is identified, it can be used to predict temperature. Scores obtained for different classes used to predict temperature. Temp is an array contain range of temperature corresponding to each classes. Scores obtained for diff erent class used for providing weights to diff erent class. if $w$ is the weight then temperature $t$ is

$$
t=\operatorname{mean}(w * t e m p)
$$

\section{EXPERIMENTS}

\section{A. Implementation details}

This is a model to identify weather condition of an image and analyze its temperature.

- Language used here is Python.

- Used IDE is Spyder.

- Anaconda distribution is used for python.

- Windows XP and 4GB RAM used.

\section{B. Evaluation Metrics}

Compared to single label classification, multilabel classification evaluation metrics is different.Predictions of each instance contain set of labels but in single label classification, prediction of each instance is a label. In multilabel classification contain three diff erent type of predictions: fully correct, partially correct and fully incorrect. In multilabel classification the method to evaluation is diff erent from single label classification. Here we consider partially correct output also. if $\mathrm{n}$ is no of instances, $Z_{i}$ is the expected output of instance and $Y_{i}$ is the actual output of instance.

\section{Exact Match Ratio}

Exact Mach Ratio(EMR), only take the output equal to the expected output. In this case partially correct output is not considered.

$$
E M R=\frac{1}{n} \sum_{i=0}^{n} Y_{i}=Z_{i}
$$

Godbole et.al(2004)[20] proposed following definitions for accuracy, precision, recall, F1-measure

\section{Accuracy}

It is defined as proportion of predicted correct labels to the total number of labels. Accuracy is

Accuracy $=\frac{1}{n} \sum_{i=n}^{n} \frac{\left|Y_{\bar{i}} \cap Z_{\bar{i}}\right|}{\left|Y_{\bar{i}} \cup Z_{\bar{i}}\right|}$

\section{Precision}

It is defined as proportion of predicted correct labels to the actual labels. Precision is

Precision $=\frac{1}{n} \sum_{i=0}^{n} \frac{\left|Y_{i} \cap Z_{i}\right|}{\left|Z_{i}\right|}$

Recall

It is defined as proportion of predicted correct labels to the total predicted labels. Recall is

Recall $=\frac{1}{n} \sum_{i=0}^{n} \frac{\left|Y_{i} \cap Z_{\bar{i}}\right|}{\left|Y_{\bar{x}}\right|}$

\section{F1-Measure}

It is defined as harmonic mean of precision and recall.

$F 1-$ Measure $=\left(\frac{\text { recall }^{-1}+\text { precision }^{-1}}{2}\right)^{-1}$

\section{Result Analysis}

It is developed as a tool for weather and temperature analysis from an image.

- In training phase, input is a dataset of 1200 images. Each class contain 200 images. Output is a python data file contain features of each images.

- In testing phase, input is a outdoor image and output is scores to each class and analyzed result of weather and temperature. Screenshots of the working of system is given in appendix section. 
In multi label classification problems output of each data instance will be set of values. In this system, if it is a sunny image the output will be set sunny. If it is a sunny and rainy image then output will be set sunny, rainy. Most of the images taken contain labels to identify their class. This is considered as expected output. In some cases manually identified their classes and used as expected output. In the training phase to train 1200 images two days needed as I used 4GB RAM. It take approximately 6 seconds to retrieve weather condition from an image.

As mentioned above, for evaluation Godbele et.al(2004)[20] method of evaluation for multilabel classification is used. EMR, accuracy, precision, recall, F1measure for the test data set is calculated. Table 1 shows the result.

Table 1. Evaluation Result

\begin{tabular}{||cc||}
\hline EVALUATION METRICS & RESULT \\
\hline \hline EMR & 0.825 \\
\hline Accuracy & 0.874 \\
\hline Precision & 0.912 \\
\hline Recall & 0.887 \\
\hline F1 measure & 0.901 \\
\hline
\end{tabular}

\section{Diff erent strategies for feature extraction}

Stage two of the project is feature extraction.For feature extraction, image is considered as whole image and clustered image. Image is divided in to clusters in clustered image and image is taken as a whole in whole image.In both cases accuracy is calculated. Figure 4 shows the bar diagram of the result obtained.Feature extraction using clustered image got better result.So clustered image is chosen for feature extraction.

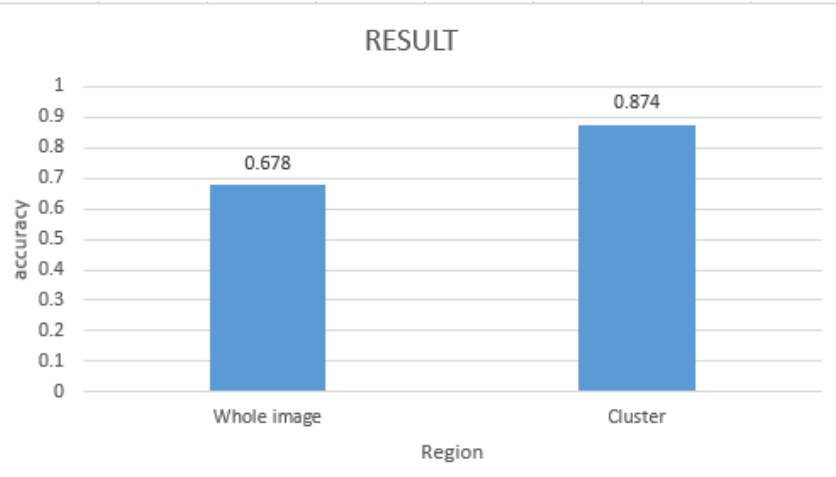

Fig.4 Different methods for feature extraction

\section{Segmentation using different clustering algorithms}

For clustering different methods are considered. Experiment is done by clustering methods like fuzzy logic, Kmeans clustering on test data set. Figure 5 shows the result.
Image segmentation using Kmeans clustering algorithm provided better result.

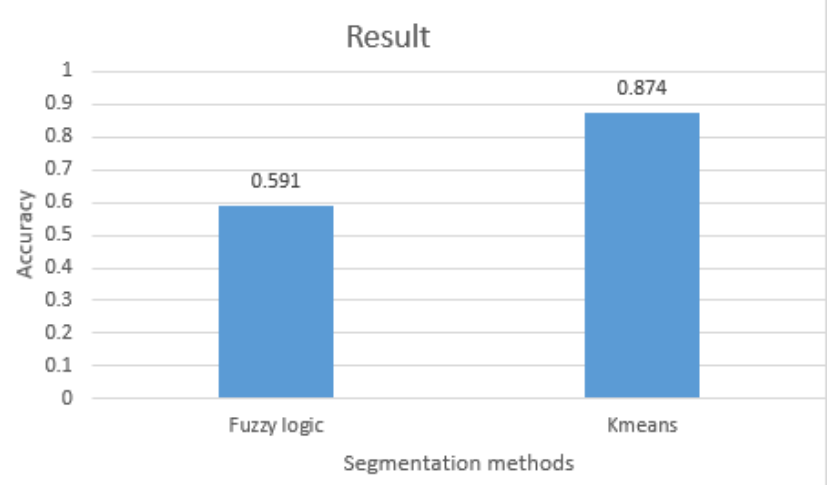

Fig. 5. Different methods for image segmentation

\section{Experiment on Two class weather dataset}

Data set used in the paper(2014)[6] divided in to sunny and cloudy classes. This data set contain 10000 images. 4000 inages from each class used for training and rest of data used as test data set. Accuracy obtained is much better than other methods. Figure below shows comparison of our method with other methods. From the table below it is clear that accuracy of non cnn methods are less.

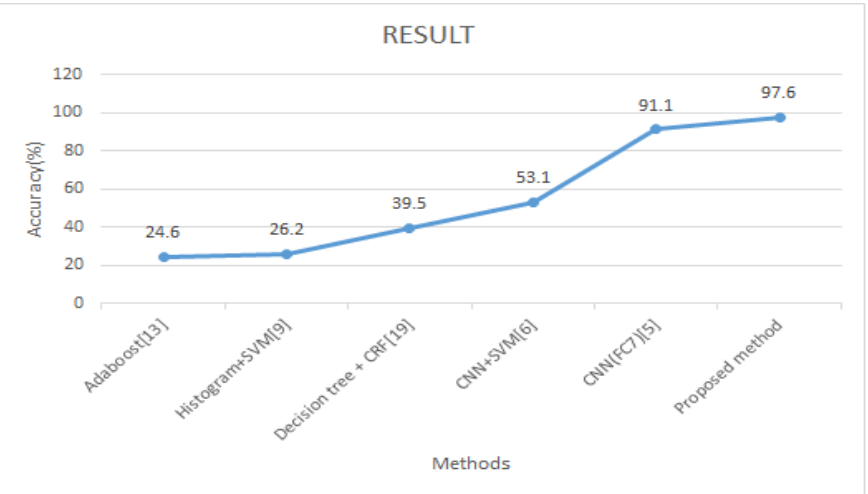

Fig. 6. Comparison on two class weather dataset

\section{SCREENSHOTS}




\section{International Journal of Engineering Applied Sciences and Technology, 2019 \\ Vol. 4, Issue 2, ISSN No. 2455-2143, Pages 59-66 \\ Published Online June 2019 in IJEAST (http://www.ijeast.com)}

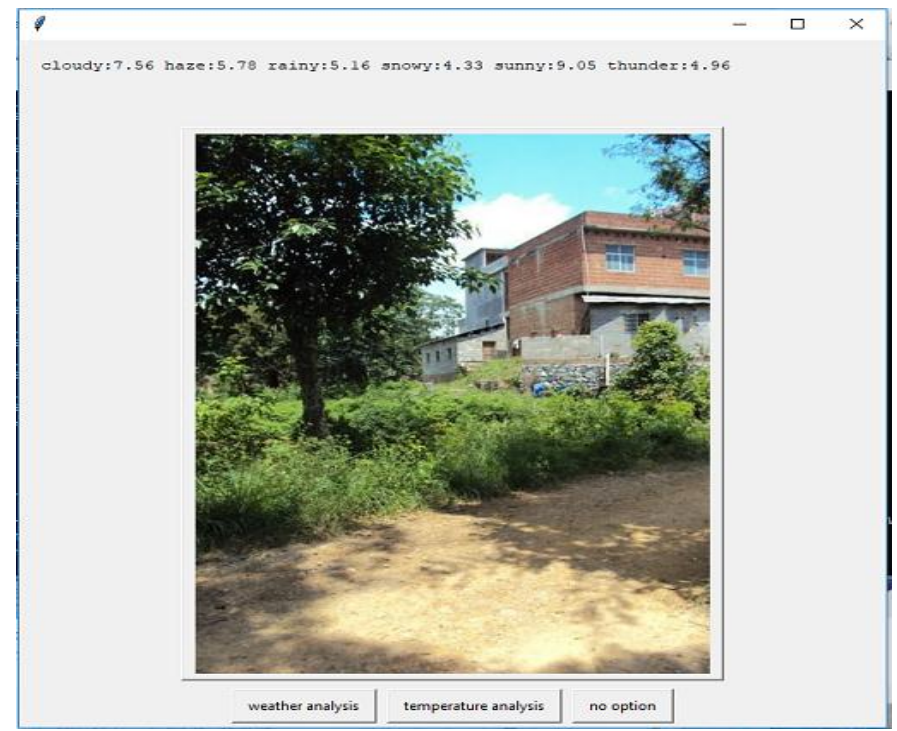

Fig.7. Weather Analysis

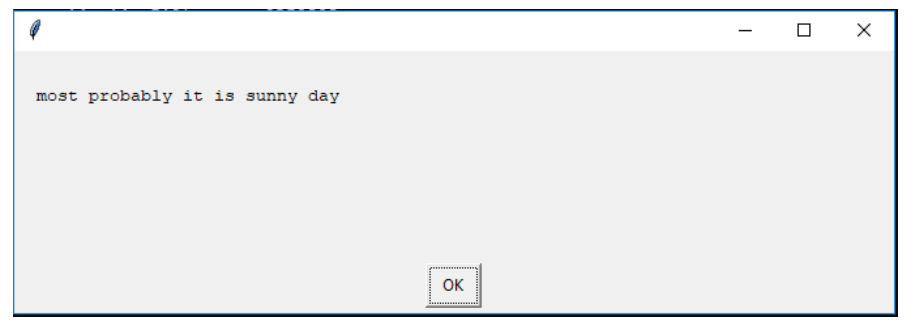

Fig.8. Weather Prediction

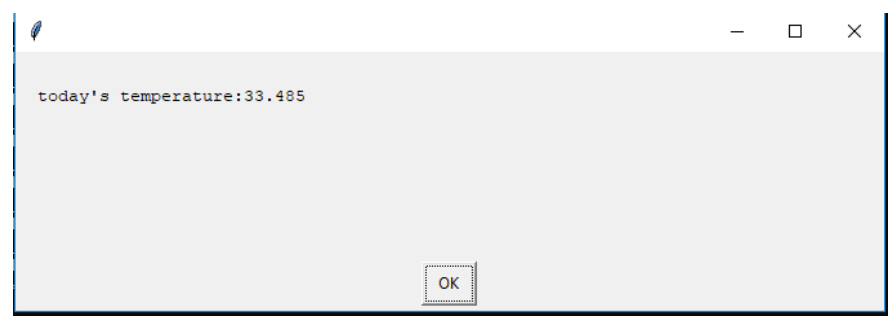

Fig.9. Temperature Prediction

\section{SCOPE OF FUTURE WORK AND APPLICABLITY}

In an image there are some regions that are not useful to determine weather. We can identify these regions during preprocessing and if it is possible to remove it before feature extraction then it can improve the performance and accuracy. Use of more sharp images in training set can improve accuracy. Image based weather recognition has many applications. One of the application is predicting weather of not reachable areas by using satellite images. Another field is robotics. It can identify the weather from the image it is captured and perform accordingly. Another application is in driving assistance. Automatic driving cars are nowadays very popular. Image based weather application helps it to identify weather without any additional hardware.

\section{CONCLUSION}

The project focused to develop a weather and temperature analysis system from images using cnn. It has two modules: weather recognition and temperature analysis. The overall working of the system is explained. In preprocessing stage, Kmeans clustering method is used for segmentation. For feature extraction VGG16 model is used as base cnn architecture. Inorder to provide coexistance of segments Euclidean distance is used. SGDC algorithm used for optimization. Decision function used to predict the scores corresponding to each classes. This scores used to predict temperature range of that image. For segmentation diff erent algorithms are considered and chose Kmeans clustering algorithm as it provides better accuracy. An experiment on Two class dataset is done. And our method had better accuracy.

\section{REFERENCES}

[1] C. Lu et.al.(2017).Two class weather classification, IEEE Transactions on pattern analysis and machine intelligence.(pg:2510-2523)

[2] Din lin et.al.(2017).RSCM: Regional Selection and Concurrency model for multiclass weather recognition, IEEE transaction on image processing.(pg:4154-4167)

[3] J. Dai et,al.(2016).Instance-aware semantic segmentation via multi-task network cascades IEEE CVPR,(pg:3150-3158).

[4] J. Yang, K. Yu, Y. Gong, and T. S. Huang.(2015).Linear spatial pyramid matching using sparse coding for image classification, IEEE ICCV(pg:.1794-1891)

[5] M. Elhoseiny, S. Huang, and A. M. Elgammal.(2015).Weather classification with deep convolutional neural networks, IEEE ICIP.(pg:3349-3353)

[6] C. Lu, D. Lin, J. Jia, and C.-K. Tang.(2014).Two-class weather classification, IEEE Conf. Comput. Vis. Pattern Recognition (CVPR).(pg:3718-3725)

[7] J. Wang, J. Yang, K. Yu, F. Lv, T. S. Huang, and Y. Gong.(2010).Locality constrained linear coding for image classification, IEEE CVPR.(pg:3360-3367)

[8] Z. Zhang and H. Ma.(2015).Multi-class weather classification on single images, IEEE ICIP.(pg:4396-4400)

[9] M. Roser and F. Moosmann.(2008).Classification of weather situations on single color images, in Proc. IEEE Intell. Veh. (pg:798-803) 
[10] Wei chu, Kai, Ali boraji.(2018).Visual weather temperature prediction,IEEE Winter conference on computer applications.(pg:234-241)

[11] A. Yu and K. Grauman.(2009).Just noticeable diff erences in visual attribute, IEEE CVPR.(pg:1794-1801)

[12] P. F. Felzenszwalb, R. B. Girshick, D. McAllester, and D. Ramanan.(2010).Object detection with discriminatively trained part-based models, IEEE Trans. Pattern Anal. Mach. Intell., vol. 32, no. 9.(1627-1645).

[13] X. Yan, Y. Luo, and X. Zheng.(2009).Weather recognition based on images captured by vision system in vehicle, in Advances in Neural Network.(pg:390-398).

[14] J. Kim, J. Frahm, and M. Pollefeys.(2008).Radiometric calibration with illumination change for outdoor scene analysis", in Proc. IEEE CVPR.(pg:1-8).

[15] C. Farabet, C. Couprie, L. Najman, and Y. LeCun. (2012).Scene parsing with multiscale feature learning, purity trees, and optimal covers, in Proc.ICML.(pg:1-8)

[16] K Simonyan et.al.(2014).Very Deep Convolutional network for large scale image recognition" ILSVRC.(14091556).

[17] J.-F. Lalonde, A. A. Efros, and S. G. Narasimhan.(2012) Estimating the natural illumination conditions from a single outdoor image, Int. J. Comput.(pg:123-145)

[18] S. Wehrwein, K. Bala, and N. Snavely.(2015).Shadow detection and sun direction in photo collections, in Proc. IEEE 3DV.(460-468).

[19] R.-E. Fan, K.-W. Chang, C.-J. Hsieh, X.-R. Wang.(2008) "LIBLINEAR: A library for large linear classification", J. Mach. Learn.Res., vol. 9, (pg:1871-1874).

[20]Godbele et.al.(2004).Discriminative method for multilabel classification. Springer.(pg:22-30)

[21] B.Alexe, T.Deselaers, andV.Ferrari.(2010). What is an object?, In CVPR.DOI:10.1109.

[22] G. Antipov, M. Baccouche, S.-A. Berrania, and J.-L. Dugelay.(2017).Eff ective training of convolutional neural networks for face-based gender and age prediction,Pattern Recognition.DOI:10.1019.

[23] W.-T.ChuandY.-L.Wu.(2016).Deep correlation features for image style classication, In Proceedings of ACM International Conference on Multimedia.(pg:402-406).

[24] Serge Belongie, Jitendra Malik, and Jan Puzicha.(2000). Shape context: A new descriptor for shape matching and object recognition,In NIPS.(pg:831-837).

[25] Richard O. Duda, Peter E. Hart, and David G. Stork.(2001) Pattern classification. Wiley, 2. ed. edition,

[26] Alex Krizhevsky, Ilya Sutskever, and Geoff rey E Hinton.(2012).Imagenet classication with deep convolutional neural networks, in Advances in neural information processing systems. 